\title{
Atomically phase-matched second-harmonic generation in a 2D crystal
}

\author{
Mervin Zhao ${ }^{1,2, \star}$, Ziliang Ye ${ }^{1,2, *}$, Ryuji Suzuki ${ }^{3,4, \star}, \mathrm{Yu} \mathrm{Ye}^{1,2}$, Hanyu Zhu ${ }^{1}$, Jun Xiao ${ }^{1}$, Yuan Wang ${ }^{1,2}$, \\ Yoshihiro Iwasa $^{3,4}$ and Xiang Zhang ${ }^{1,2,5}$
}

\begin{abstract}
Second-harmonic generation (SHG) has found extensive applications from hand-held laser pointers to spectroscopic and microscopic techniques. Recently, some cleavable van der Waals (vdW) crystals have shown SHG arising from a single atomic layer, where the SH light elucidated important information such as the grain boundaries and electronic structure in these ultra-thin materials. However, despite the inversion asymmetry of the single layer, the typical crystal stacking restores inversion symmetry for even numbers of layers leading to an oscillatory SH response, drastically reducing the applicability of vdW crystals such as molybdenum disulfide $\left(\mathrm{MoS}_{2}\right)$. Here, we probe the SHG generated from the noncentrosymmetric $3 R$ crystal phase of $\mathrm{MoS}_{2}$. We experimentally observed quadratic dependence of second-harmonic intensity on layer number as a result of atomically phase-matched nonlinear dipoles in layers of the $3 \mathrm{R}$ crystal that constructively interfere. By studying the layer evolution of the $\mathrm{A}$ and $\mathrm{B}$ excitonic transitions in $3 \mathrm{R}-\mathrm{MoS}_{2}$ using SHG spectroscopy, we also found distinct electronic structure differences arising from the crystal structure and the dramatic effect of symmetry and layer stacking on the nonlinear properties of these atomic crystals. The constructive nature of the SHG in this 2D crystal provides a platform to reliably develop atomically flat and controllably thin nonlinear media.
\end{abstract}

Light: Science \& Applications (2016) 5, e16131; doi:10.1038/lsa.2016.131; published online 26 August 2016

Keywords: atomically thin; phase-matching; second-harmonic generation; 3R-MoS 2

\section{INTRODUCTION}

The optical second-harmonic generation (SHG) is the nonlinear frequency doubling process that has been demonstrated over 50 years ago in quartz ${ }^{1}$ with numerous applications, including those in microscopic ${ }^{2}$ and spectroscopic techniques ${ }^{3}$. However, as SHG arises from $\chi^{(2)}$, strong SH signals may only be observed from noncentrosymmetric crystals. As the demand for smaller and thinner nonlinear media becomes higher in next-generation optoelectronic devices, great efforts have been devoted to solve the phase-mismatch problems in nonlinear crystals. While techniques such as quasi-phase matching ${ }^{4}$ or creating zero-index metamaterials ${ }^{5}$ are viable methods, reducing the dimensionality of such nonlinear media to two dimensions would eliminate the requirement for phase matching as the crystal thickness is far below the coherence length. Naturally, two-dimensional (2D) van der Waals (vdW) crystals may serve as an ideal candidate for such materials, as the $\mathrm{SH}$ polarization is confined within a single molecular layer that may be exfoliated and isolated from a bulk crystal. Unfortunately, the vdW crystals that exhibit nonlinearity within a single layer typically do not produce $\mathrm{SH}$ signals when inversion symmetry is restored in their multilayer counterparts.
Group VI transition metal dichalcogenides (TMDCs), such as $\mathrm{MoS}_{2}, \mathrm{WS}_{2}, \mathrm{MoSe}_{2}$ and $\mathrm{WSe}_{2}$ have gained prominent attention due to many unique emerging properties once these materials are thinned down to single or a few atomic layers. Rich physical properties, such as the emerging photoluminescence ${ }^{6,7}$, excellent transistor on-off ratio ${ }^{8}$, optical valley polarization ${ }^{9-11}$, large exciton and trion binding energies $^{12,13}$, exciton electroluminescence ${ }^{14}$, ambipolar transport ${ }^{15}$, superconductivity ${ }^{16}$ and piezoelectricity ${ }^{17,18}$ have been demonstrated in this 2D crystal system. Many of these properties arise as a result of the layer-dependent evolution of crystal symmetry.

Thus far, the most commonly studied bulk crystal phase of $\mathrm{MoS}_{2}$ is the $2 \mathrm{H}$ polytype, with two $\mathrm{S}-\mathrm{Mo}-\mathrm{S}$ unit cells of trigonal prismatic coordination and space group $D_{6 h}\left(P \sigma_{3} / m m c\right)$ (Figure 1a). The symmetry of the $2 \mathrm{H}$ phase dictates that the flakes with odd-numbered layers are noncentrosymmetric, retaining a net nonlinear dipole, while centrosymmetric even layers do not. This net nonlinear dipole in oddlayered TMDCs (along with $h$-BN) has enabled the probing of SHG from 2D crystals due to the finite second-order nonlinearity $\left(\chi^{(2)} \neq 0\right)^{19-23}$. The SH signal from single-layer TMDCs has been utilized to study the crystal orientation and grain boundaries ${ }^{24}$, while also enabling a spectroscopic method to study the excitonic structure ${ }^{25}$.

${ }^{1}$ Department of Mechanical Engineering, NSF Nanoscale Science and Engineering Center, University of California, Berkeley, CA 94720, USA; ${ }^{2}$ Materials Sciences Division, Lawrence Berkeley National Laboratory, Berkeley, CA 94720, USA; ${ }^{3}$ Quantum-Phase Electronics Center (QPEC) and Department of Applied Physics, The University of Tokyo, Tokyo 113-8656, Japan; ${ }^{4}$ RIKEN Center for Emergent Matter Science (CEMS), Wako 351-0198, Japan and ${ }^{5}$ Department of Physics, King Abdulaziz University, Jeddah 21589, Saudi Arabia

*These authors contributed equally to this work

Correspondence: X Zhang, Email: xiang@berkeley.edu

Received 21 September 2015; revised 7 March 2016; accepted 7 March 2016; accepted article preview online 14 March 2016 


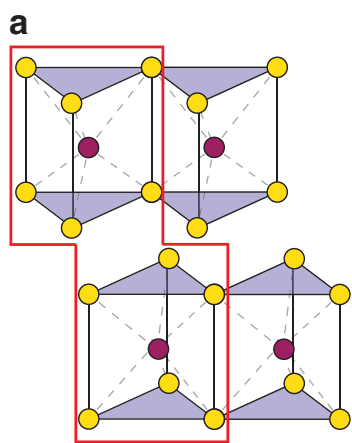

b



OS
Side view
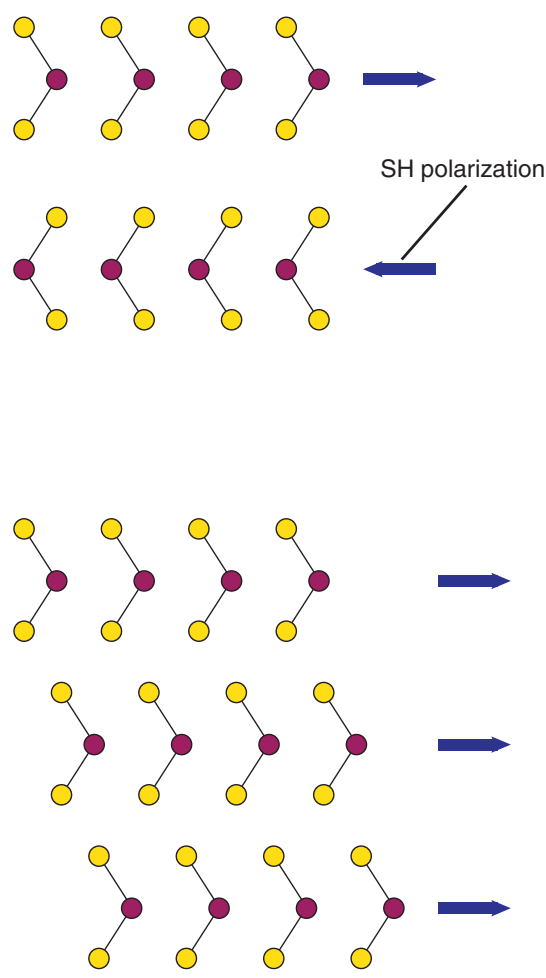

Figure 1 Crystal structure of $2 \mathrm{H}$ and $3 \mathrm{R}-\mathrm{MoS}_{2}$. (a) Schematic of the crystal structure of $2 \mathrm{H}-\mathrm{MoS}_{2}$ (two layer unit cell outlined in red) with Mo atoms in maroon and $\mathrm{S}$ atoms in yellow. The Mo atoms are hexagonally packed within each layer and trigonal prismatically coordinated with $\mathrm{S}$ atoms on the top and bottom. The side projection shows the flipped orientation of each layer and the anti-parallel orientation of the SH dipoles that allow for SHG. (b) Crystal structure of $3 \mathrm{R}-\mathrm{MoS}_{2}$ (three layer unit cell outlined in red). Individual layers are identical to the $2 \mathrm{H}$ structure, however the unit cell and bulk crystal are noncentrosymmetric. The layers are oriented in such a way that the dipoles are parallel allowing for constructive interference of the SHG light.

This all-optical technique allows for non-destructive crystal structure studies, and has advantages over transmission electron microscopy and atomic-force microscopy (AFM) based techniques due to the simplicity of sample preparation. Unfortunately, the crystal symmetry constrains the SHG in the $2 \mathrm{H}$ phase to noncentrosymmetric oddnumbered layers. Coupled with a typically decaying $\mathrm{SH}$ intensity as odd layers become thicker due to the finite absorption ${ }^{19}$, the applicability of the SHG from $2 \mathrm{H}-\mathrm{MoS}_{2}$ is significantly reduced.

Aside from the $2 \mathrm{H}$ phase, $\mathrm{MoS}_{2}$ coexists in the equally stable bulk noncentrosymmetric $3 \mathrm{R}$ polytype. The orientation of the atomic layers in the $3 \mathrm{R}$ phase, of space group $C_{3 v}(R 3 m)$, leads to parallel in-plane nonlinear dipoles (Figure $1 \mathrm{~b}$ ), making the crystal an excellent candidate for a cleavable, atomically flat, phase-matched 2D nonlinear crystal. The single-layers of these two crystal phases are identical (' $1 \mathrm{H}$ ', group $D_{3 h}$ ). However, adjacent layers of the $2 \mathrm{H}$ are mirrored to restore the inversion symmetry, while the layers in the $3 \mathrm{R}$ phase retain the same orientation but shift along the in-plane direction. The alignment of the in-plane dipole of each $3 \mathrm{R}$ layer allows for an atomic phase match and leads to the constructive interference of the $\mathrm{SH}$ polarization in the thin-film limit below the coherence length, where we expect the intensity to scale quadratically with the layer number. In the bulk crystalline forms (over $10 \mu \mathrm{m}$ thick), the SHG of the $3 \mathrm{R}$ and $2 \mathrm{H}$ has been studied over a decade ago showing the observable and nearly zero SHG, respectively ${ }^{26}$. While the SHG observed in chemically grown $\mathrm{MoS}_{2}$ spirals ${ }^{27}$ as well as folded $\mathrm{MoS}_{2}$ bilayers ${ }^{28-30}$ yield some insight, there have not yet been reports on the nature of the SHG from the 3R phase in the atomically phase-matched regime, crucial for the development of ultra-thin nonlinear optical devices.

\section{MATERIALS AND METHODS}

\section{Growth and characterization of $\mathrm{MoS}_{2}$ polytypes}

Bulk $3 \mathrm{R}$ and $2 \mathrm{H}-\mathrm{MoS}_{2}$ crystals were grown using chemical-vapor transport technique with $\mathrm{Cl}_{2}$ and $\mathrm{I}_{2}$ as the carrier gas, respectively ${ }^{31}$. Crystals ( $3 \mathrm{R}$ and $2 \mathrm{H}-\mathrm{MoS}_{2}$ ) were mechanically exfoliated onto a fused quartz substrate. Layer number assignment was determined using a combination of photoluminescence imaging, Raman spectroscopy (Horiba LabRAM HR Evolution, Edison, NJ, USA) 31,32 $^{3}$ and AFM (Dimension 3100, Digital Instruments, Santa Barbara, CA, USA) (Supplementary Fig. S3).

\section{Optical measurements}

The SH measurement was conducted with a reflection geometry with the excitation normal to the sample at room temperature. We used a Ti-Sapphire oscillator (Chameleon, Coherent, Glasgow, Scotland) to pump an optical parametric oscillator (Chameleon Compact OPO, Coherent, Berlin, Germany) with $80 \mathrm{MHz}$ repetition to achieve tunable near-infrared wavelength from 1000 to $1550 \mathrm{~nm}$ in $200 \mathrm{fs}$ pulses. The laser is directed to a microscope with a scanning stage. The average laser power was kept at $90 \mathrm{~mW}$ for the $3 \mathrm{R}$ excitation and a similar power for the $2 \mathrm{H}$ excitation at each excitation energy before 
entering the microscope. The laser is focused by a $63 \times$ objective with a numerical aperture of 0.75 to a spot size of around $2 \mu \mathrm{m}$. The reflected signal is directed to an $805 \mathrm{~nm}$ short-pass dichroic mirror and two band-pass filters (315-725 and 350-700 nm) to eliminate the reflected pump beam. The signal is detected using a photomultiplier tube with a detection range of $300-720 \mathrm{~nm}$ (H7422-40, Hamamatsu, Japan). Each SH intensity is normalized using the transmission of our optical setup.

\section{RESULTS AND DISCUSSION}

We probed the layer dependence of the SHG from the $3 \mathrm{R}$ and $2 \mathrm{H}-\mathrm{MoS}_{2}$ across a broad energy range, and found that the $\mathrm{SH}$ intensity in the $3 \mathrm{R}$ polytype scales quadratically with layer number up to six layers. By mapping out the $\mathrm{SH}$ signal with energies from 1.77 to $2.30 \mathrm{eV}$, two strong $\mathrm{SH}$ resonances are found. Compared with linear optical absorption measurements, we showed the resonances are due to the $\mathrm{A}$ and $\mathrm{B}$ excitonic transitions in the $\mathrm{MoS}_{2}$, arising from spin-orbit coupling and interlayer interactioninduced splitting. We show that the SHG in a $3 \mathrm{R}$ crystal allows for the nonlinear probing of the bulk excitons beyond an odd-layer limit, while also providing an alternative all-optical method of layer number determination.

The optical image of the $3 \mathrm{R}$ crystal with the layer number overlaid is presented in Figure 2a. In this crystal, we assign the few-layers 1-4 and 6 by Raman spectroscopy and AFM (Supplementary Figs. S1-S3). This can be immediately compared with the respective $\mathrm{SH}$ image pumped at an energy of $1.14 \mathrm{eV}$ in Figure $2 \mathrm{~b}$. We can easily distinguish between the $3 \mathrm{R}$ and $2 \mathrm{H}$ polytypes through the image produced through the $\mathrm{SH}$ scanning. The $\mathrm{SH}$ intensity produced by the $3 \mathrm{R}$ crystal is highest in the thickest of layers, while the SHG from a $2 \mathrm{H}$ crystal is only noticeable in the odd-numbered layers (Supplementary Fig. S4) ${ }^{20}$.

Following a similar approach to the reported SHG in single-layer $\mathrm{MoS}_{2}{ }^{(\text {refs. } 19,21)}$ and assuming weak interlayer coupling, the inclusion of the parallel $\mathrm{SH}$ polarizations in the $3 \mathrm{R}$ crystal from $N$ layers leads to the summation of $N$ polarizations. Thus, we predict a crystal of $N$ layers to produce a $\mathrm{SH}$ intensity given by:

$$
I(2 \omega)=|E(2 \omega)|^{2}=C^{2} N^{2} \chi_{\mathrm{MoS}_{2}}^{(2)}(\omega) \cos ^{2}\left(3 \phi+\phi_{0}\right)
$$

where $C$ is a proportionality constant describing the local field factors, and $\phi$ and $\phi_{0}$ describe the angle of the laser polarization and initial crystallographic orientation, respectively. This predicts the observed intensity to quadratically vary with respect to the layer number within the thin-film limit. However, as the linear absorption in $\mathrm{MoS}_{2}$ may become non-negligible in our $\mathrm{SH}$ photon energy range from 1.77 to $2.30 \mathrm{eV}$, the attenuation from reabsorption is expected to be nontrivial, particularly as the layer numbers increase. In addition, changes in the refractive index $\mathrm{x}^{33-35}$ at the fundamental and $\mathrm{SH}$ energies play a role in the attenuation of the experimental SHG (Supplementary Fig. S6).

The observed $\mathrm{SH}$ layer dependence of the $3 \mathrm{R}$ and $2 \mathrm{H}$ crystal polytypes a SH energy of $1.82 \mathrm{eV}$ are shown in contrast in Figure 3a. We have normalized the $\mathrm{SH}$ intensity of different layer numbers to that of the respective single-layer intensity, resulting in the larger deviations as the layer number increased. Representative data before the normalization is shown in Supplementary Fig. S7. The SHG from the $3 \mathrm{R}$ crystal is distinct from the oscillating signal of the $2 \mathrm{H}$ crystal, with only observable SHG in odd layers. Using the log-log relationship, we mapped out the $N$ layers' power dependence, $\alpha$, across the entire bandwidth (Figure $3 \mathrm{~b}$ ). We observed a quadratic dependence in good agreement with our model of the SHG intensity produced by the $3 \mathrm{R}$ crystal from energies of 1.77 to $1.83 \mathrm{eV}$, as well as from 1.93 to $1.98 \mathrm{eV}$. Two clear dips are observed in the power dependence at energies of around 1.87 and $2.07 \mathrm{eV}$, corresponding to excitonic enhancements in the single-layer intensity.

In order to understand the range of the predicted $N^{2}$ dependence, we performed $\mathrm{SH}$ spectroscopy on the $3 \mathrm{R}$ crystal using excitation energies ranging from 0.88 to $1.22 \mathrm{eV}$. From the SHG spectra of single-layer and six-layer $3 \mathrm{R}-\mathrm{MoS}_{2}$ (Figure 4a), we observe two dramatic enhancements in the $\mathrm{SH}$ intensity. These two enhancements at around 1.85 and $2.00 \mathrm{eV}$ correspond well to the known $\mathrm{A}$ and $\mathrm{B}$ excitons, also observed in the linear absorption (Supplementary Fig. S5), correspond directly to the dips observed in the power law. The two resonances are the excitonic transitions originated from the conduction band and two split valence bands at the K-point of the Brillouin zone.

Due to the atomically thin nature of the sampled crystal, we expect that the deviations from the $N^{2}$ dependence arise primarily from the sensitivity of the SHG to small shifts in resonant energy. At high $\mathrm{SH}$ energies (roughly beyond $2 \mathrm{eV}$ ) the linear absorption remains non-negligible, effectively attenuating the $\mathrm{SH}$ intensity as the thickness increases through reabsorption. Furthermore, an obvious red shift in the $\mathrm{A}$ and $\mathrm{B}$ excitons were observed when changing from a single layer
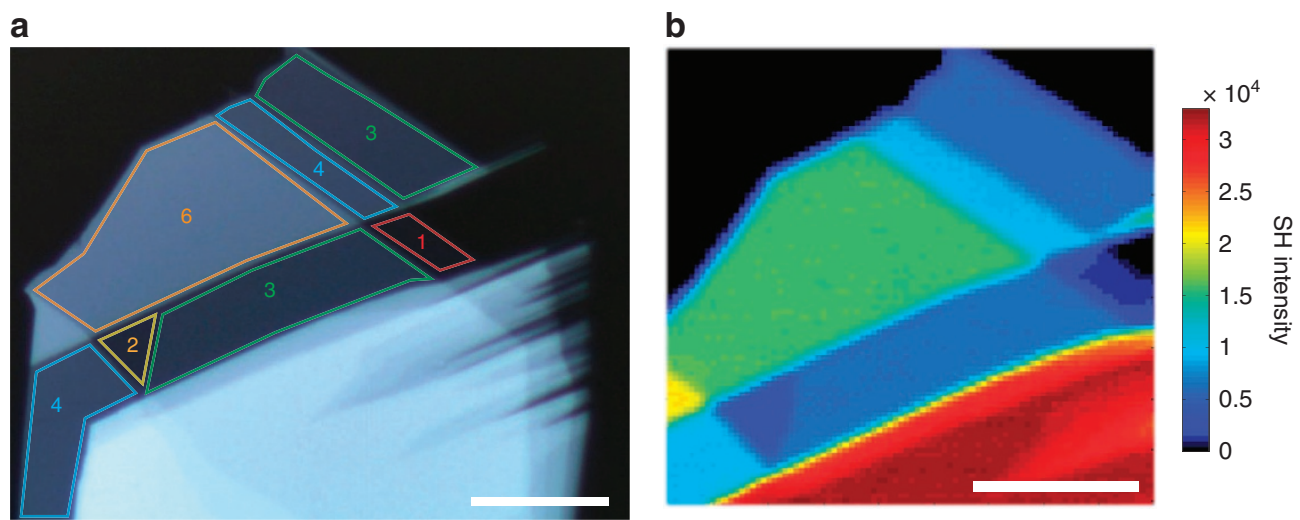

Figure 2 Optical and SH microscopy images of 3R-MoS 2 . (a) Optical image of the 3R-MoS 2 crystal exfoliated onto a fused quartz substrate. Here, we overlay the layer number on top of the corresponding thickness, which was verified via using a combination of photoluminescence mapping, Raman spectroscopy and atomic-force microscopy. (b) Image produced via scanning and gathering the SH light produced by the 3R-MoS 2 (excitation energy of $1.14 \mathrm{eV}$ ). We see that the SH intensity in increases with layer thickness, shown in a with the single-layer having the lowest intensity. The strongest emissions at the left and bottom of the crystal (given by yellow and red) correspond to layer numbers greater than six. Scale bars shown in $\mathbf{a}$ and $\mathbf{b}$ correspond to $15 \mu \mathrm{m}$. 

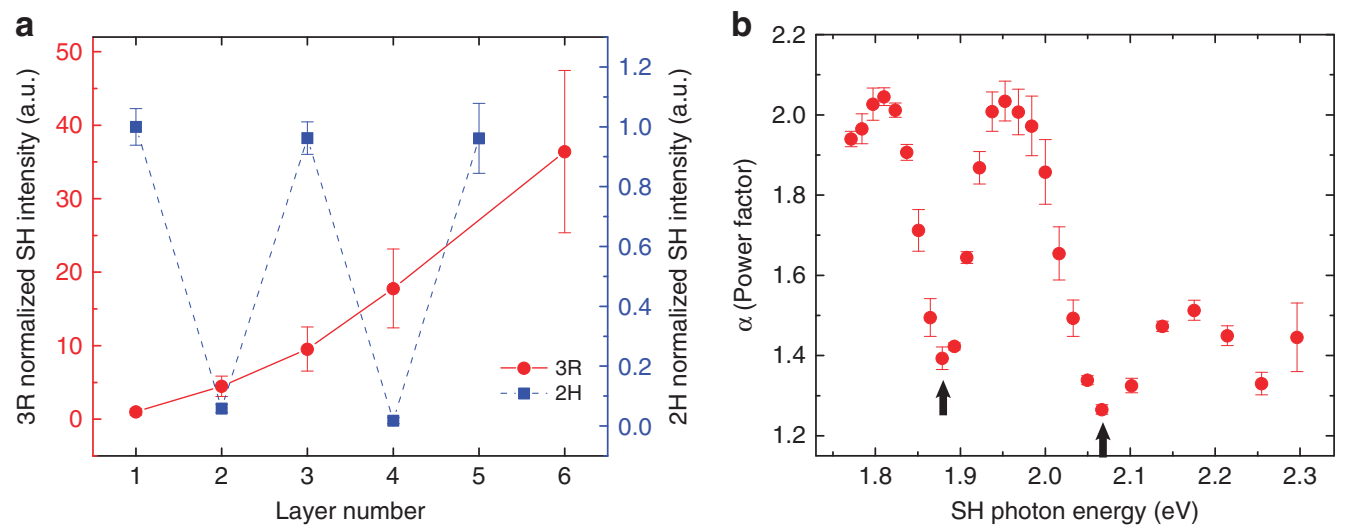

Figure 3 Layer dependence and scaling of the SH light. (a) SH intensity of the $3 \mathrm{R}$ and $2 \mathrm{H}-\mathrm{MoS}_{2}$ normalized to the respective single-layer intensity at a SH energy of $1.81 \mathrm{eV}$. The dependence of the $\mathrm{SH}$ intensity is roughly squared with relation to the layer number in the $3 \mathrm{R}$ crystal, while it oscillates with layer number in the $2 \mathrm{H}$ crystal. The left axis corresponds to the $3 \mathrm{R}$ intensity, while the right axis corresponds to the $2 \mathrm{H}$ intensity. (b) Power law ( $N^{\alpha}$, where $N$ is the layer number) derived from the log-log relationship in data such as a across energies from 1.77 to $2.3 \mathrm{eV}$ to determine the range of the expected phasematching relationship, $N^{2}$. We note the energies below $1.85 \mathrm{eV}$ and energies between 1.9 and $2.0 \mathrm{eV}$ correspond to scaling accordingly to $N^{2}$. Two dips marked by arrows correspond to the $\mathrm{A}(1.85 \mathrm{eV})$ and $\mathrm{B}(2.05 \mathrm{eV})$ excitons of the single-layer $\mathrm{MoS}_{2}$. The enhancement due to the excitons in the single layer decreases the expected scaling of the SHG across the other layers. The deviations above $2.1 \mathrm{eV}$ are noted to arise from the broader linear absorption at energies above the B exciton.

to six layers (Figure 4a), due to electronic changes as a result of interlayer interactions. These exciton shifts dramatically influence the observed layer dependence due to the high enhancements of the $\mathrm{SH}$ intensity around the exciton resonances. The $\mathrm{SH}$ resonances produced by the A (B) exciton has a red shift of 10 (50) $\mathrm{meV}$ from layers one to six. These energy offsets causes the SH enhancements of each layer to occur at different energies and reveals why the quadratic dependence is observed at the lowest probed energies, as well as select energies between the two resonant enhancements, where SH absorption and excitonic enhancements do not strongly affect the SHG. Considering the finite absorption even at lower energies, we have observed a very close to $N^{2}$ dependence up to six layers. Beyond six layers the SH light is attenuated due to a combination of the reabsorption and the phase accumulation from the refractive index change. These two factors become significant even at ten layers, as there is roughly a $20 \%$ decrease in the idealized SH intensity (Supplementary Fig. S6).

$\mathrm{SH}$ spectroscopy was also performed on the $2 \mathrm{H}$ crystal in the same energy range using the same experimental setup (Figure $4 \mathrm{~b}$ ). It was noted that many of the works on SHG in $2 \mathrm{H}-\mathrm{MoS}_{2}{ }^{\text {(refs. 19-21) }}$ used an excitation around $1.5 \mathrm{eV}$, corresponding to $\mathrm{SH}$ energies of around $3 \mathrm{eV}$. These energies fall into the range of the broad exciton feature at the $\Gamma$-point ${ }^{21}$. As a result, large $\mathrm{SH}$ enhancement produced from the single-layer $2 \mathrm{H}-\mathrm{MoS}_{2}$, as well as the high sensitivity of the $\mathrm{SH}$ intensity produced by the odd-numbered layers was observed. Using $\mathrm{SH}$ spectroscopy, we observed that the overall SHG between the odd layers are of comparable magnitude, with the even layers showing no detectable SHG, as expected. The A and $\mathrm{B}$ exciton features are observed here as well. In our experimental range, the single-layer SHG is enhanced most by the B exciton, while the A exciton seems to enhance the odd layers to a comparable degree. As with the 3R sample, both SHG peaks are red-shifted as the odd layer number increases, though to a lesser extent.

We expect that our results provide more insight into the electronic evolution of the $3 \mathrm{R}$ crystal with increasing layer number. Theoretical calculations show that the single-layer valence-band splitting (around $180 \mathrm{meV}$, Figure 4c) at the K-point arises solely from spin-orbit interactions, while the splitting in two and more layers of the $2 \mathrm{H}$ crystal is primarily a result of the interlayer-hopping interactions. Due to the symmetry, the wave functions form interlayer bonding and anti-bonding states ${ }^{36,37}$. In contrast, calculated wave functions at the K-point of the 3R crystal are localized, suppressing interlayer hopping. The effects of which are apparent (Figure 4c), the valence-band splitting from a $1 \mathrm{H}$ single-layer to a $3 \mathrm{R}$ two layer decreases by $20 \mathrm{meV}$, while the $2 \mathrm{H}$ splitting is nearly constant regardless of layer number. The lack of interlayer hopping suggests that the origin of the valenceband splitting in the bulk $3 \mathrm{R}$ crystal arises from the unique crystal stacking geometry, resulting in an inequality of the electrostatic potential energy at the $\mathrm{MoS}_{2}$ prisms in the two layers ${ }^{29,31,38}$. We probe a splitting of $135 \mathrm{meV}$ in six layers, nearly $50 \mathrm{meV}$ smaller than the splitting found in the $2 \mathrm{H}$ bulk crystal, in agreement with the reported bulk splitting found from linear absorption and angleresolved photoemission spectroscopy ${ }^{31}$. Our findings are summarized in the simplified band structure in Figure $4 \mathrm{~d}$. We were able to experimentally measure the layer by layer evolution of the valenceband splitting in the $3 \mathrm{R}$ phase for the first time, using $\mathrm{SH}$ spectroscopy.

\section{CONCLUSIONS}

In conclusion, we have measured the atomically phase-matched SHG arising from a $3 \mathrm{R}-\mathrm{MoS}_{2}$ crystal with layer numbers up to six. The SHG intensity scales with the layer number, $N$, as $N^{2}$ in the range of $\mathrm{SH}$ energies of $1.77-1.84$ and $1.95-1.98 \mathrm{eV}$. The $\mathrm{SH}$ spectrum from 1.77 to $2.30 \mathrm{eV}$ showed two strong enhancements corresponding to the $\mathrm{A}$ and $\mathrm{B}$ excitons in $\mathrm{MoS}_{2}$, which cause the deviations in the $\mathrm{N}^{2}$ dependence, even though the $\mathrm{SH}$ photons are phase-matched. These findings enable the probing of the $3 \mathrm{R}-\mathrm{MoS}_{2}$ excitons with $\mathrm{SH}$ spectroscopy in the bulk, as well as provide an all-optical tool for the determination of layer number in the $3 \mathrm{R}$ polytype of TMDCs. We demonstrated that $3 \mathrm{R}-\mathrm{MoS}_{2}$ is a cleavable nonlinear crystal that generates $\mathrm{SH}$ light in all of its crystalline layers. Coupled with the potential of tuning the excitonic states and thus $\mathrm{SH}$ intensities through electric gating ${ }^{13,39}$, the $3 \mathrm{R}$ crystal polytype is an exceptional material choice in the development of ultra-thin nonlinear optical devices. The 3R polytype of TMDCs along with recently engineered twisted 
a

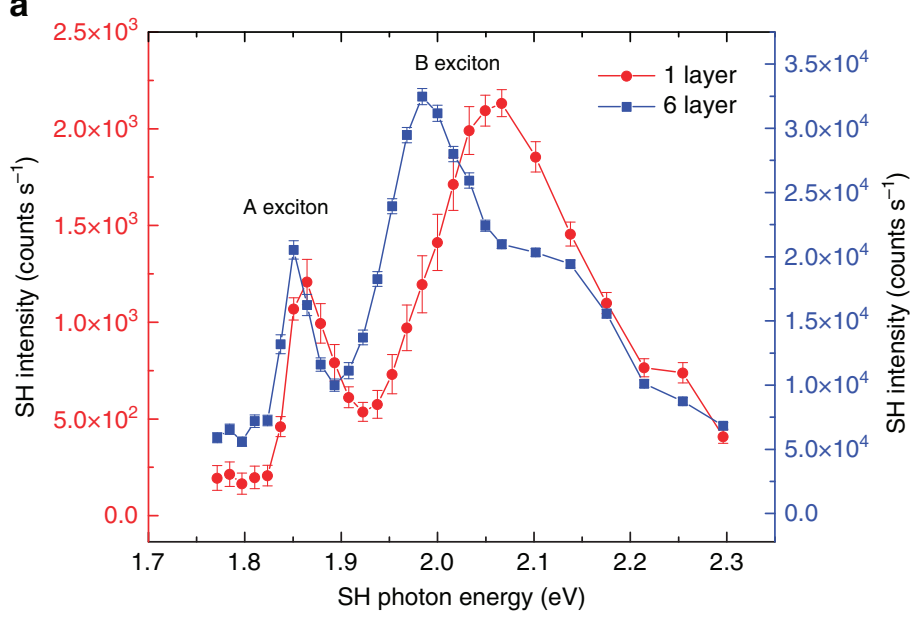

c



b

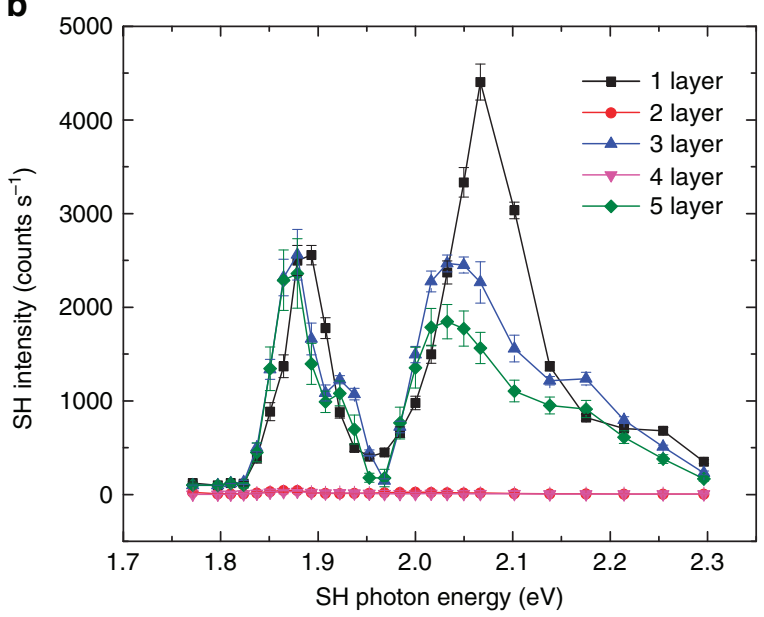

d

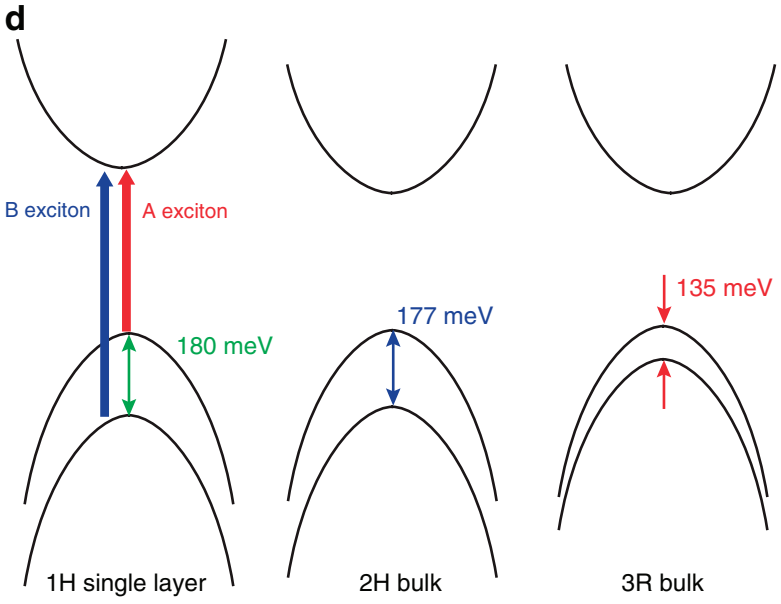

Figure $4 \mathrm{SH}$ spectroscopy. (a) SH spectra of the 3R-MoS 2 . The two peaks occurring at around 1.85 and $2.0 \mathrm{eV}$ correspond to the A and $\mathrm{B}$ excitons of $\mathrm{K}$-point transitions in $\mathrm{MoS}_{2}$. The six-layer intensity is one order larger than the single-layer intensity. Increasing the layer number decreases both exciton energies, with the B exciton experiencing a much larger shift (roughly $50 \mathrm{meV}$ ). (b) $\mathrm{SH}$ spectra of the $2 \mathrm{H}-\mathrm{MoS}_{2}$, showing a similar response as the $3 \mathrm{R}$ crystal. Only odd layers show SH intensities with comparable amplitudes in the layers. The two and four-layered intensities are overlapped. (c) The A and B exciton energy differences, corresponding to the valence-band splitting, of the $3 \mathrm{R}$ and $2 \mathrm{H}$ crystal are plotted. The $2 \mathrm{H}$ exciton splitting remains close to $180 \mathrm{meV}$ up to five layers, while the $3 \mathrm{R}$ exciton splitting dramatically decreases to $130 \mathrm{meV}$ at six layers (which agrees to the bulk value obtained from linear absorption). (d) Simplified electronic band structure showing transitions from the valence band to the excitonic band, derived from the SH spectroscopy data at the $\mathrm{K}$-point of the single layer and bulk $2 \mathrm{H}$ and $3 \mathrm{R}$ phases. The energy of the A exciton decreases from single layer to bulk in both crystal phases by around $10 \mathrm{meV}$ (exemplified in a lower excitonic band of the bulk crystals), however the valence-band splitting is dramatically different, which effects the large difference between the B exciton energy in the two phases. This splitting difference is due to the different stacking geometries and suppressedinterlayer hopping, resulting in an inequality of the electrostatic potential energy at the $\mathrm{MoS}_{2}$ prisms in the two layers.

bilayers ${ }^{28-30}$ and CVD grown $\mathrm{MoS}_{2}$ spirals $^{27}$ show promise for an additional avenue in controlling the rich physical properties of vdW materials through the manipulation of interlayer symmetries.

\section{CONFLICT OF INTEREST}

The authors declare no conflict of interest.

\section{ACKNOWLEDGEMENTS}

This research was supported by the Office of Naval Research (ONR) MURI program under Grant No.N00014-13-1-0649 and the National Science Foundation (NSF, Grant No. EFMA-1542741). MZ acknowledges support from NSF Graduate Research Fellowship (Grant No.DGE 1106400). YI and RS acknowledge support from the Grants-in-Aid for Specially Promoted Research (No.25000003) by the Japan Society for the Promotion of Science (JSPS). RS was supported by JSPS through a research fellowship for young scientists and through the Materials Education Program for the Future Leaders in Research, Industry and Technology (MERIT).

\section{AUTHOR CONTRIBUTIONS}

$\mathrm{MZ}, \mathrm{ZY}$ and RS designed the experiment; RS grew the $\mathrm{MoS}_{2}$ crystals; HZ prepared the quartz substrate for the experiment; $M Z$ and $Z Y$ conducted the optical experiments; MZ, ZY, YY, JX and HZ analyzed the data. XZ guided the research. All authors contributed to the discussion and preparation of the manuscript.

\footnotetext{
1 Franken PA, Hill AE, Peters CW, Weinreich G. Generation of optical harmonics. Phys Rev Lett 1961; 7: 118-119.
}

2 Campagnola PJ, Loew LM. Second-harmonic imaging microscopy for visualizing biomolecular arrays in cells, tissues and organisms. Nat Biotechnol 2003; 21: 1356-1360. 
3 Eisenthal KB. Liquid interfaces probed by second-harmonic and sum-frequency spectroscopy. Chem Rev 1996; 96: 1343-1360.

4 Fejer MM, Magel GA, Jundt DH, Byer RL. Quasi-phase-matched second harmonic generation: tuning and tolerances. IEEE J Quant Electron 1992; 28: 2631-2654.

5 Suchowski H, O'Brien K, Wong ZJ, Salandrino A, Yin X et al. Phase mismatch-free nonlinear propagation in optical zero-index materials. Science 2013; 342: 1223-1226.

6 Mak KF, Lee C, Hone J, Shan J, Heinz TF. Atomically thin $\mathrm{MoS}_{2}$ : a new direct-gap semiconductor. Phys Rev Lett 2010; 105: 136805.

7 Splendiani A, Sun L, Zhang YB, Li TS, Kim J et al. Emerging photoluminescence in monolayer $\mathrm{MoS}_{2}$. Nano Lett 2010; 10: 1271-1275.

8 Radisavljevic B, Radenovic A, Brivio J, Giacometti V, Kis A. Single-layer $\mathrm{MoS}_{2}$ transistors. Nat Nano 2011; 6: 147-150.

9 Mak KF, He KL, Shan J, Heinz TF. Control of valley polarization in monolayer $\mathrm{MoS}_{2}$ by optical helicity. Nat Nano 2012; 7: 494-498.

10 Cao T, Wang G, Han WP, Ye HQ, Zhu CR et al. Valley-selective circular dichroism of monolayer molybdenum disulphide. Nat Commun 2012; 3: 887.

11 Zeng HL, Dai JF, Yao W, Xiao D, Cui XD. Valley polarization in $\mathrm{MoS}_{2}$ monolayers by optical pumping. Nat Nano 2012; 7: 490-493.

12 Ye ZL, Cao T, O'Brien K, Zhu HY, Yin XB et al. Probing excitonic dark states in singlelayer tungsten disulphide. Nature 2014; 513: 214-218.

13 Mak KF, He KL, Lee C, Lee GH, Hone J et al. Tightly bound trions in monolayer MoS $_{2}$. Nat Mater 2013; 12: 207-211.

14 Ye Y, Ye ZL, Gharghi M, Zhu HY, Zhao M et al. Exciton-dominant electroluminescence from a diode of monolayer MoS $_{2}$. Appl Phys Lett 2014; 104: 193508.

15 Zhang YJ, Ye JT, Matsuhashi Y, Iwasa Y. Ambipolar MoS 2 thin flake transistors. Nano Lett 2012; 12: 1136-1140.

16 Ye JT, Zhang YJ, Akashi R, Bahramy MS, Arita R et al. Superconducting dome in a gatetuned band insulator. Science 2012; 338: 1193-1196.

17 Zhu HY, Wang Y, Xiao J, Liu M, Xiong SM et al. Observation of piezoelectricity in freestanding monolayer MoS 2 . Nat Nanotechnol 2015; 10: 151-155.

18 Wu WZ, Wang L, Li YL, Zhang F, Lin L et al. Piezoelectricity of single-atomic-layer MoS 2 for energy conversion and piezotronics. Nature 2014; 514: 470-474.

$19 \mathrm{Li} \mathrm{YL}$, Rao Y, Mak KF, You YM, Wang SY et al. Probing symmetry properties of few-layer $\mathrm{MoS}_{2}$ and h-BN by optical second-harmonic generation. Nano Lett 2013; 13: 3329-3333.

20 Kumar N, Najmaei S, Cui QN, Ceballos F, Ajayan P et al. Second harmonic microscopy of monolayer MoS2. Phys Rev B 2013; 87: 161403.

21 Malard LM, Alencar TV, Barboza APM, Mak KF, de Paula AM. Observation of intense second harmonic generation from $\mathrm{MoS}_{2}$ atomic crystals. Phys Rev B 2013; 87: 201401.

22 Janisch $\mathrm{C}$, Wang $\mathrm{YX}$, Ma D, Mehta N, Elias AL et al. Extraordinary second harmonic generation in tungsten disulfide monolayers. Sci Rep 2014; 4: 5530.

23 Zeng HL, Liu GB, Dai JF, Yan YJ, Zhu BR et al. Optical signature of symmetry variations and spin-valley coupling in atomically thin tungsten dichalcogenides. Sci Rep 2013; 3: 1608.

24 Yin X, Ye Z, Chenet DA, Ye Y, O'Brien $\mathrm{K}$ et al. Edge nonlinear optics on a $\mathrm{MoS}_{2}$ atomic monolayer. Science 2014; 344: 488-490.
25 Wang G, Marie X, Gerber I, Amand T, Lagarde D et al. Giant enhancement of the optical second-harmonic emission of $\mathrm{WSe}_{2}$ monolayers by laser excitation at exciton resonances. Phys Rev Lett 2015; 114: 097403.

26 Wagoner GA, Persans PD, Van Wagenen EA, Korenowski GM. Second-harmonic generation in molybdenum disulfide. J Opt Soc Am B 1998; 15: 1017-1021.

27 Zhang LM, Liu KH, Wong AB, Kim J, Hong XP et al. Three-dimensional spirals of atomic layered $\mathrm{MoS}_{2}$. Nano Lett 2014; 14: 6418-6423.

28 Liu KH, Zhang LM, Cao T, Jin CH, Qiu D et al. Evolution of interlayer coupling in twisted molybdenum disulfide bilayers. Nat Commun 2014; 5: 4966.

29 Jiang T, Liu HR, Huang D, Zhang S, Li YG et al. Valley and band structure engineering of folded $\mathrm{MoS}_{2}$ bilayers. Nat Nanotechnol 2014; 9: 825-829.

30 van der Zande AM, Kunstmann J, Chernikov A, Chenet DA, You YM et al. Tailoring the electronic structure in bilayer molybdenum disulfide via interlayer twist. Nano Lett 2014; 14: 3869-3875.

31 Suzuki R, Sakano M, Zhang YJ, Akashi R, Morikawa D et al. Valley-dependent spin polarization in bulk $\mathrm{MoS}_{2}$ with broken inversion symmetry. Nat Nano 2014; 9: 611-617.

32 Lee C, Yan HG, Brus LE, Heinz TF, Hone J et al. Anomalous lattice vibrations of singleand few-layer $\mathrm{MoS}_{2}$. ACS Nano 2010; 4: 2695-2700.

33 Shen CC, Hsu YT, Li LJ, Liu HL. Charge dynamics and electronic structures of monolayer $\mathrm{MoS}_{2}$ films grown by chemical vapor deposition. Appl Phys Express 2013; 6: 125801.

34 Zhang $\mathrm{H}$, Ma YG, Wan $\mathrm{Y}$, Rong $\mathrm{X}$, Xie Z et al. Measuring the refractive index of highly crystalline monolayer $\mathrm{MoS}_{2}$ with high confidence. Sci Rep 2015; 5: 8440.

$35 \mathrm{Li} \mathrm{W}$, Birdwell AG, Amani M, Burke RA, Ling $X$ et al. Broadband optical properties of large-area monolayer CVD molybdenum disulfide. Phys Rev B 2014; 90 : 195434.

36 Cheiwchanchamnangij T, Lambrecht WRL. Quasiparticle band structure calculation of monolayer, bilayer, and bulk MoS 2 . Phys Rev B 2012; 85: 205302.

37 Molina-Sánchez A, Sangalli D, Hummer K, Marini A, Wirtz L. Effect of spin-orbit interaction on the optical spectra of single-layer, double-layer, and bulk $\mathrm{MoS}_{2}$. Phys Rev B 2013; 88: 045412 .

38 Akashi R, Ochi M, Bordács S, Suzuki R, Tokura Y et al. Two-dimensional valley electrons and excitons in noncentrosymmetric 3R-MoS2. Phys Rev Appl 2015; 4: 014002.

39 Seyler KL, Schaibley JR, Gong P, Rivera P, Jones AM et al. Electrical control of second-harmonic generation in a WSe 2 monolayer transistor. Nat Nano 2015; 10: 407-411.

(c) (1) This work is licensed under a Creative Commons AttributionBY NC SA NonCommercial-ShareAlike 4.0 International License. The images or other third party material in this article are included in the article's Creative Commons license, unless indicated otherwise in the credit line; if the material is not included under the Creative Commons license, users will need to obtain permission from the license holder to reproduce the material. To view a copy of this license, visit http:// creativecommons.org/licenses/by-nc-sa/4.0/

Supplementary Information for this article can be found on the Light: Science \& Applications' website (http://www.nature.com/lsa). 